\title{
A Review on the Underwater Image Restoration and Enhancement Methods
}

Siaw-Lang Wong ${ }^{1}$, Raveendran Paramesran ${ }^{2 *}$, Akira Taguchi ${ }^{3}$ and Ong Seng Huat ${ }^{4}$

\author{
${ }^{1}$ Faculty of Computing and Information Technology, Tunku Abdul Rahman University College, 53300 Kuala Lumpur, \\ Malaysia \\ ${ }^{2}$ Institute of Computer Science and Digital Innovation, UCSI University, 56000 Kuala Lumpur, Malaysia \\ ${ }^{3}$ Faculty of Information Technology, Tokyo City University, Tokyo, 158-8557 Japan \\ ${ }^{4}$ Faculty of Business and Management, UCSI University, 56000 Kuala Lumpur, Malaysia
}

\begin{abstract}
Light scattering, as well as light absorption in the water, often cause underwater images to be hazy, poorly contrasted, and dominated by either green or blue colour cast. In this paper, we review some of the state-of-the-art approaches in which specifically designed to enhance the quality of the acquired images. These approaches are able to eliminate the color cast and haziness on the images as well as to improve the image colourfulness and contrast. The characteristics of each of the developed approaches are highlighted, and their performances are evaluated both subjectively and objectively by the quality assessment methods.
\end{abstract}

Keywords: colour cast; image enhancement; underwater image processing; image restoration

\section{INTRODUCTION}

Nowadays, there is a keen interest in knowing what lies underwater. Modern technology has impressively changed human's view of underwater photography. Underwater cameras are much easier to use, and housing manufacturers have given scuba divers great options to capture underwater images. This field has made the importance of the underwater images in scientific missions such as monitoring marine species, accessing geological or biological underwater environments and searching for shipwrecks. In order to achieve these missions, there is a need to acquire clear underwater images as well as understand the science of light and colour in the water medium. Capturing a clear underwater image is exciting yet challenging. The fundamental physics of the light transmission in the water medium must be investigated first in order to deal with the underwater image processing. Light plays an essential role in all capturing devices. Without perceiving the incoming light reflected from the object, the capturing devices produce the images with all dark pixels (Abdul Ghani, 2015).
Underwater images are poorly visible caused by two primary sources: light scattering and absorption of light by water (Chiang \& Chen, 2012). The water medium's physical properties cause degradation effects that do not exist in atmospheric images. When light travels through the water instead of air, special techniques and equipment are required for underwater photography because water has very distinct characteristics than air. Water is an 800-times denser substance than air. The light interacts with the water molecules and suspended particles as soon as it penetrates the water (Anthoni, 2012).

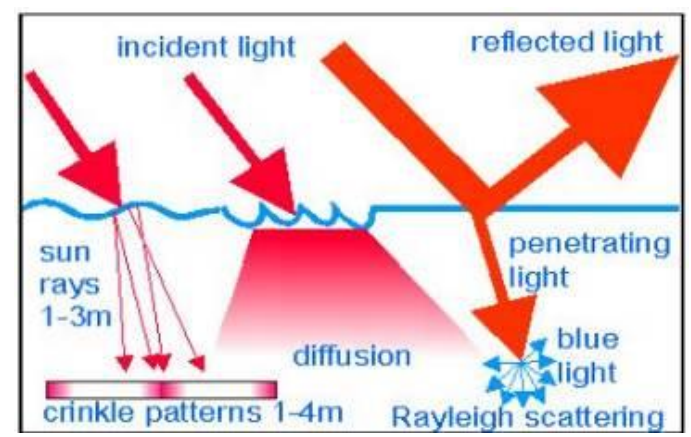

Figure 1. Water surface effects (Anthoni, 2012) 
As illustrated in Figure 1, when the light coming from optically less dense medium air and entering the denser medium water, the light is partially reflected back while partially penetrates the water. Moreover, the light produces crinkle patterns or spreads spontaneously in omnidirectional depending on the water shape. The volume of light reflected upward was influenced by the sea condition and sun height. A rough sea absorbs more light while a calm sea brings more reflection (Anthoni, 2012). When travels in the water, light is attenuated exponentially since it is scattered and deflected by the water particles for multiple times before reaching the camera sensor. All these, as a result, caused the underwater images to be hazy and poorly contrasted. Besides that, haze is also caused by suspended particles such as minerals, sands and plankton that present in the oceans, rivers and lakes (Chiang \& Chen, 2012).

The decisions and techniques range from purely postprocessing methods to novel hardware configurations depending greatly on the imaging system, location, and goals of the photographer. The idea of what is "interesting" in an image is exclusively guided by the opinions and objectives of the observer (Kaeli, 2013). For shallow and naturally-lit imagery, exciting results have been obtained using polarizing filters (Schechner \& Karpel, 2004) and dehazing algorithms (Fattal, 2008; Treibitz \& Schechner, 2009). For increasing the grayscale contrast, homomorphic filtering (Singh et al., 2007; Ghani \& Isa, 2015) and histogram equalization methods (Hitam et al., 2013; Abdul Ghani \& Mat Isa, 2014, 2017) are useful and effective to be implemented. In highly turbid environments, exotic lighting techniques (Gupta et al., 2008; Jaffe, 2010; Gorman, 2011) have been employed. For restoring the image colour, methods may range from the simple white balancing (Chambah et. al., 2004; Wong et al., 2018) to Markov Random Fields (Torres-Mendez \& Dudek, 2005), and even the coloured strobes (Vasilescu et al., 2011). Lastly, the enhancement method fuses different colour spaces (Iqbal et al., 2007; Ao \& Ma, 2018) have produced quite significant results.

The rest of the paper is organized as follows. In Section 2, the primary principle of image formation introduced by McGlamery (1980) and Jaffe (1990) is elaborated. Next, the state-of-the-art image enhancement methods developed by previous researchers are presented and critically reviewed in
Section 3. Section 4 presents the experimental studies and the comparative findings among state-of-the-art methods. Finally, the paper is concluded in Section 5 .

\section{UNDERWATER IMAGE FORMATION}

To understand the underwater phenomenon, the primary underwater image formation will be studied. The theoretical fundamental of underwater image formation is firstly introduced by McGlamery (1980) where he pointed out the theoretical basis of the optical image formation concept. Then Jaffe (1990) expanded the concept and applied it to build various subsea image acquisition systems.

According to Jaffe (1990), the propagation of light in underwater, which is considered electromagnetic waves in isotropic homogenous media, should obey the wave equations expressed through Maxwell's equations. The two processes by which light interacts with the water column are scattering and absorption. Scattering refers to any deflection from a straight-line propagation direction, while absorption is the power loss when light passes through the water medium, depending on the medium's refraction index. Deflections can be triggered in the underwater environment by the particles of size similar to the wavelengths of moving light (diffraction) or by the particulate matter with a refractive index different from that of the water (refraction).

Next, most researchers adopt Jaffe-McGlamery's image formation concept, where the underwater image described three components as a linear superposition. As illustrated in Figure 2, an experiment on underwater image involves locating the transition of light from the light source to the camera. The light that received by the camera is comprised of three components, which are

1. Direct component $E_{d}$ - refers to the light directly reflected by the object that has not been scattered in the water,

2. Forward-scattered component $E_{f}$ - refers to the light reflected by the scattered object at a minor angle, as well as

3. Backscatter component $E_{b}$ - refers to the light reflected by the objects that do not appear on the target scene but enter the camera, such as floating particles. 


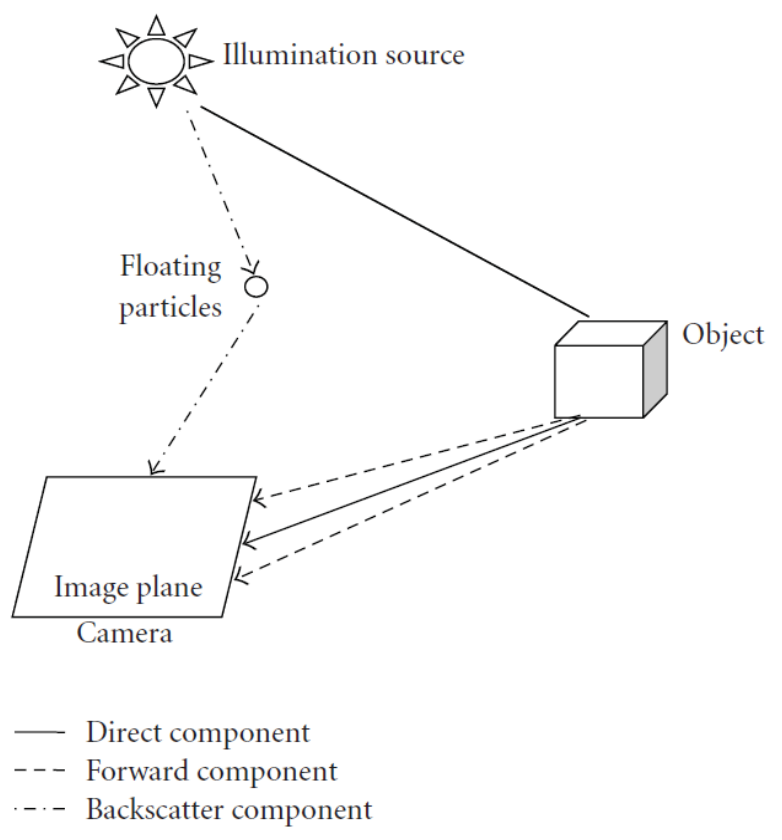

Figure 2. The components of underwater imaging, which are direct component (represented as straight line), forwardscattered component (represented as dashed line), and backscatter component (represented as dash-dot line)

(Schettini \& Corchs, 2010)

In order to calculate the direct component $E_{d}$, it is necessary to consider three components: the geometric optics of the camera, the spherical spreading of the reflected wave, and the medium attenuation between the camera and the reflectance map. Via the relationship of convolution, the forward-scattered component $E_{f}$ can then be determined from the direct component. On the other hand, regardless of the small angle approximation, the measurement of the backscatter component $E_{b}$ is the most computationally costly, because the backscattered light reaches the camera sensor from a wide angle range (Jaffe, 1990). Finally, the total irradiance $E_{T}$ can be written mathematically as:

$$
E_{T}=E_{d}+E_{f}+E_{b}
$$

The details derivation of each of the components can refer to Jaffe (1990).

Underwater image formation is essential information needed by the researchers to analyze the underwater image as this information indirectly describes the parameters and elements, which are contributed toward the problems of underwater image. As previously described, light is the main component in the underwater image that affects the overall quality of the captured underwater image. The effects of light propagation underwater caused many problems in the underwater image such as colour loss, colour cast and poor contrast. Moreover, insufficient light source and floating particles also produce a blurry image that reduces the image sharpness.

All these underwater problems have attracted more attention from researchers as the demand for underwater applications has increased. For that reason, underwater image processing becomes one of the most important fields of researches these days. Researchers have developed different state-of-the-art methods to reduce or remove these problems as presented in the following section.

\section{UNDERWATER IMAGE PROCESSING}

It is always desirable for an underwater image to appear as if it were taken in the air, for pre-processing of automated classification. Underwater image processing can be approached from two separate contexts: image restoration methods or image enhancement methods (Schettini \& Corchs, 2010). Both of these approaches have almost identical goals of recovering and improving the underwater image quality, where the resulting images will have improved contrast, saturation, intensity and brightness.

\section{A. Underwater Image Restoration Methods}

Image restoration targets to restore a distorted image using the degradation model and original image formation. This approach is complicated as it requires many model parameters, for instance, attenuation and diffusion coefficients that characterize the water turbidity and depth approximation of a given subject in the scene.

They have been many techniques and methods developed by researchers all around the world to restore underwater images. Chao and Wang (2010) used the image dehazing method to recover the original clarity of the underwater images. By using the Dark Channel Prior (DCP), the turbid water depth can be predicted by assuming that most local patches in the atmospheric images contain certain pixels with very small intensities in at least one colour channel. Mathematically, the model commonly used to explain the haze image formation is: 


$$
I(x)=J(x) t(x)+B(1-t(x))
$$

where $I(x)$ is the intensity observed, $J(x)$ is the intensity that is going to recover, $t(x)$ is the transmission map and ${ }_{B}$ is the background light. The dark channel for an image $J(x)$ is defined as:

$$
J_{\text {dark }}(x)=\min _{c \in r, g, b}\left(\min _{y \in \Omega(x)}\left(J_{c}(y)\right)\right)
$$

where $J_{c}$ is a colour channel of $J(x)$ and $\Omega(x)$ is a local patch centered at $x$. According to the dark channel theory that introduced by He, Sun and Tang $(2009,2011)$, the intensity of $J_{\text {dark }}$ is low and tends to be zero, if $J(x)$ is an image in the pure water. The final scene radiance $J(x)$ is recovered by:

$$
J(x)=I(x) \cdot \frac{B}{\max \left(t(x), t_{0}\right)+B}
$$

where a typical value of $t_{0}$ is o.1. Though the original colors are not recovered, the proposed method has significantly improved the clarity of the images.

At the same time, Carlevaris-Bianco et al. (2010) also presented a dehazing algorithm to eliminate the effects of light scattering in the underwater images. To measure the scene depth, they proposed an efficient prior that uses the significant difference in attenuation among the three image colour channels in water. Then, the scene depth estimation can be used to minimize the spatially varying impact of haze in the image. In addition, the dehazing process has also produced an up-to-scale scene depth map.

Besides that, Chiang and Chen (2012) presented an efficient technique for improving the underwater images using DCP algorithm to compensate the attenuation difference along the propagation path as well as to take into account the effect of the likely presence of an artificial light source. Once the depth map is determined, the foreground and background are segmented within a scene, and comparisons are made between the foreground and background light intensities to decide if an artificial light source is engaged during the image acquisition process. Upon compensating the effect of artificial light, the haze phenomenon and difference in attenuation of wavelength are adjusted along the underwater propagation path to the camera. The water depth in the image scene is then determined based on the residual energy ratios of multiple color channels present in the background light. Depending on the amount of attenuation proportional to each light wavelength, correction for the colour change is performed to recover the colour balance. Image after the dehazing and colour change compensation introduced through the propagation path $d(x)$ can be formulated as:

$$
J_{\lambda}(x)=\frac{I_{\lambda}(x)-\left(1-\operatorname{Nrer}(\lambda)^{d(x)}\right) \cdot B_{\lambda}}{\operatorname{Nrer}(\lambda)^{d(x)}}, \lambda \in\{r, g, b\}
$$

where $J_{\lambda}(x)$ is the scene radiance at the point $x, I_{\lambda}(x)$ is the camera-captured image, $\operatorname{Nrer}(\lambda)$ is the normalized residual energy ratio, $B_{\lambda}$ is the homogenous background light and $\lambda$ is the light wavelength.

Moreover, Drews-Jr et al. (2013) proposed a methodology for estimating the transmission in underwater environments which consists of DCP adaptation, a statistical prior based on the properties of the image obtained in the natural outdoor scenes. The methodology, called Underwater Dark Channel Prior (UDCP), basically assumes that the blue and green colour channels are the source of underwater visual information, allowing substantial improvements over the existing DCP-based methods. It is believed that the primary source of visual information underwater is assumed to be the blue and green colour channels, and their UDCP has provided a greater transmission approximation of underwater scenes than the conventional DCP.

Galdran et al. (2015) suggested a Red Channel method where, as predicted for underwater images, colours associated with short wavelengths are recovered, leading to a recovery of the contrast lost. The Red Channel method can be defined as a variance of the DCP method used for atmospherically degraded images when exposed to haze. They also include a general technique for identifying artificially illuminated areas within an underwater scene, preventing the presence of colour artifacts due to the wrong estimation of depth in them. They also suggested a twofold evaluation for their experimental tests. First, to assess the image contrast improvement, they used a metric introduced by Hautière et al. (2008) to calculate the visibility enhancement on fog-degraded images. Second, to assess the 
quality of the recovered colors, they proposed the use of three specific metrics: visibility recovery coefficient $e$, restoration quality coefficient $r$ and saturation coefficient $\lambda$. After image restoration, the metrics adjust well to the problematic of assessing the quality of underwater colours.

Next, Emberton et al. (2015) introduced a single image dehazing method for underwater images with novel veiling light and transmission estimation measures that deal with issues arising from bright objects. They used features to rate an image's regions hierarchically and choose the most likely candidate for veiling light. To find optimum transmission values for the areas that suffer from over-saturation, a regionbased approach is employed. They also identify background regions by superpixel segmentation and clustering and adjust the transmission values to prevent artifacts in these regions.

Li, Guo, Chen, et al., (2016) developed a physics-based approach for the estimation of global background light by using quad-tree subdivision and graph-based segmentation, and for the prediction of medium transmission map based on the theory of minimal information loss and underwater imaging optical properties. Further, Li, Guo, Cong, et al. (2016) suggested another underwater image enhancement approach that involves an underwater image dehazing algorithm based on the theory of minimal information loss and contrast enhancement method based on a histogram distribution prior. Their suggested approach consists of two key components: underwater image dehazing and contrast enhancement. The flowchart is shown in Figure 3.

Subsequently, Peng and Cosman (2017) have introduced a new restoration method based on both image blurriness and light absorption, offering a more accurate background light and depth estimation. First, in an underwater image, they picked the background light from blurred regions. The depth map and the transmission maps are then obtained to restore scene radiance based on the background light.

Akila and Varatharajan (2018) presented an optimizationbased algorithm for dehazing underwater images with an improved fuzzy intensification method. The enhanced fuzzy membership feature is derived for every colour channel. Then,

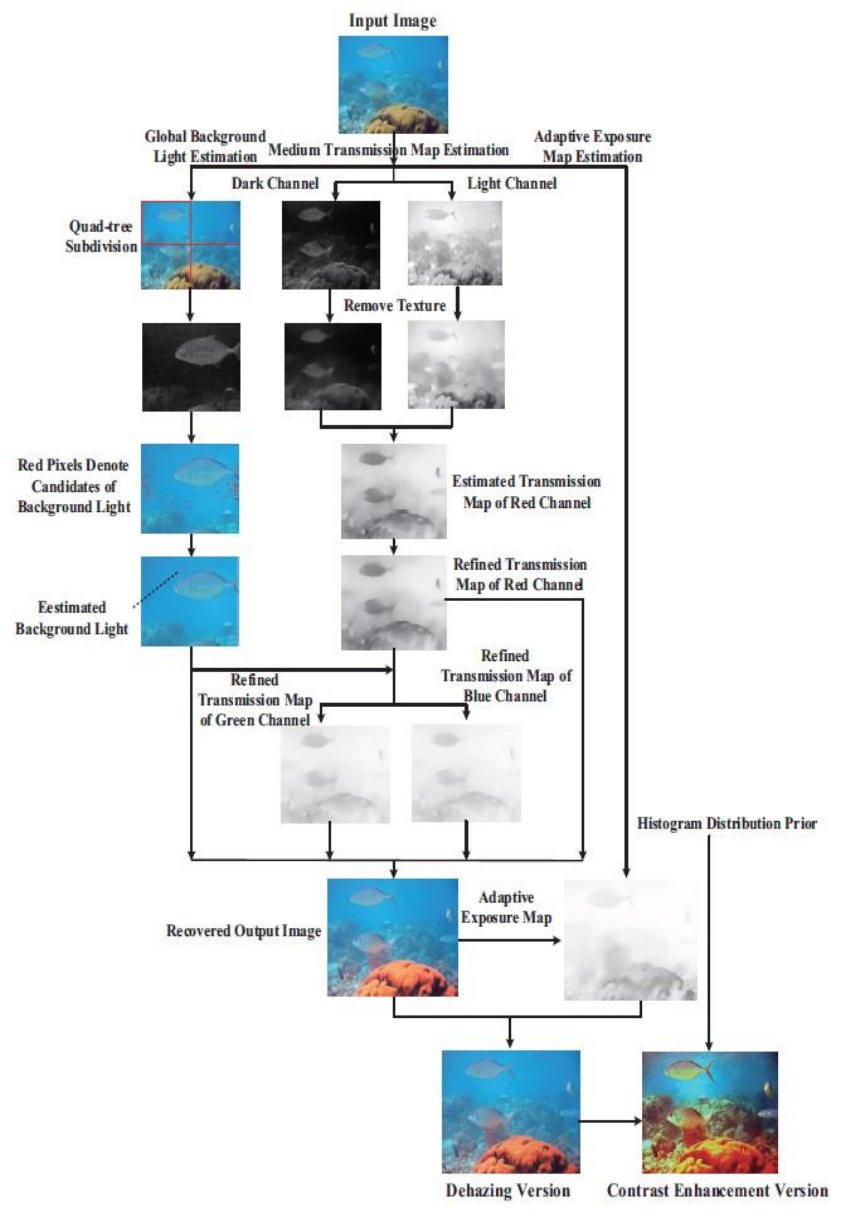

Figure 3. The flowchart of the Li, Guo, Cong, et al., (2016) method, where it composed of two main parts: underwater image dehazing and underwater image contrast enhancement

the fuzzy-based pixel intensification correction is performed for each channel to eliminate the haze and improve the colour and visibility. For red channel alone, the fuzzy histogram equalization is applied when the input image has the maximum pixel values of red channel.

Next, Xie et al. (2018) used the DCP model and underwater backscatter model for underwater marine science recognition and robot navigation. The proposed approach solved the over-exposure and over-dark problems created by the models of original DCP and backscatter. The module of environmental recognition is introduced, which can choose the most appropriate image enhancement parameters according to the similar underwater environments and then precisely enhance a variety of underwater environments. The overview of the framework is shown in Figure 4. 


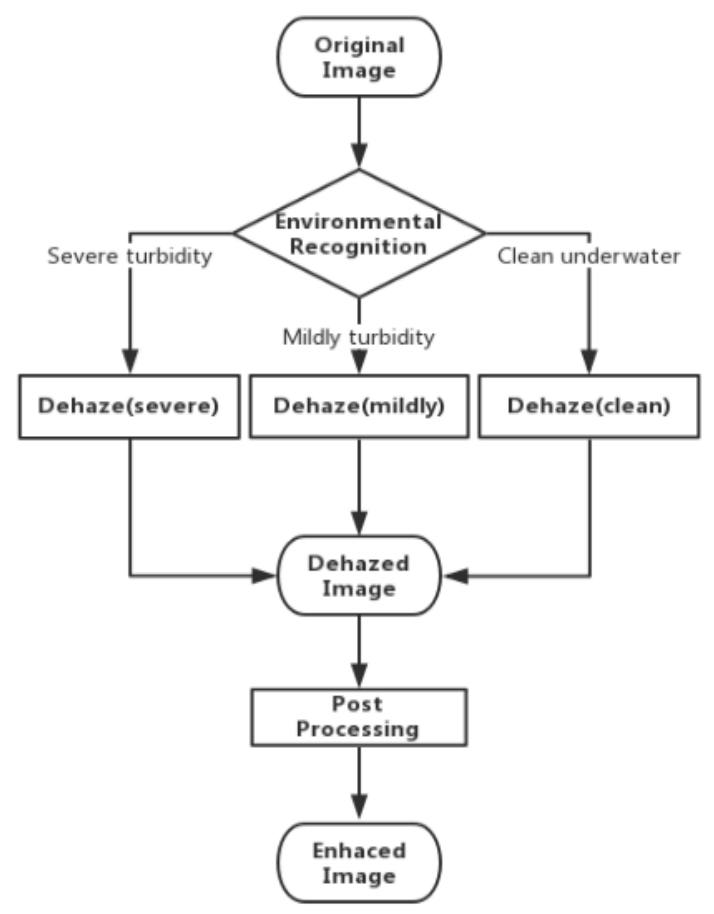

Figure 4. The framework of Xie et al., (2018) method

From the methods mentioned above, the resultant underwater image shows a notable improvement by applying restoration methods. However, the restoration methods require more input data in order to produce better resultant underwater images. Moreover, the input data used in the restoration processes are predicted and modelled, which involved many equations, parameters and coefficients. The data processing usually requires high computational consumption as these methods involve complexity mathematically. Thus, image enhancement techniques may provide better choices to improve underwater image quality without considering the initial image conditions. In the next subsection, the discussion will focus on image enhancement techniques, including automatic white balance and histogrambased image enhancement.

\section{B. Underwater Image Enhancement Methods and Color Correction}

Alternatively, image enhancement is a non-specific method used to enhance the images visual quality or the visual perception for human viewers (Gonzalez \& Woods, 2008). Image enhancement technique is one of the techniques that are popularly used for underwater image processing. Unlike image restoration, image enhancement methods are typically more straightforward and quicker than the methods for image restoration as they render the image creation process to be totally abstract and no prior knowledge of the environment is needed. The approaches mainly involved the images modifications in the spatial domain, leading to improved image color and contrast.

The development of the image enhancement methods for underwater image processing had gained significant interest in recent decades. Based on the Von Kries theorem, Iqbal et al. (2010) have revised the Red and Green colour channels to minimize the colour cast. A contrast correction is being introduced to the image pixels in $R G B$ colour space. Then, the image histograms are stretched at one or two ends, taken at the original histogram's lowest and highest points, $0.2 \%$ and $99.8 \%$, respectively. The red colour channel, which is the lowest intensity colour channel, the contrast stretching is towards the upper end; while the Green colour channel, which is the mid-intensity colour channel, the contrast stretching is at both ends; and lastly the blue colour channel, which is the main colour cast, the contrast stretching is towards the lower end. After that, the image is transformed into HSI colour space, where the $S$ and $I$ channels are stretched to both the upper and lower ends of the histogram. The overall contrast performances of the enhanced images for both approaches have been successfully improved. However, overall observations reveal that in some regions, the images are under-enhanced, especially the regions are dark.

Next, Hitam et al. (2013) proposed methods for underwater images called CLAHE-Mix. This method is designed to minimize the substantial noise presented by CLAHE method to facilitate the subsequent processing of underwater images. The CLAHE is first used in the $R G B$ colour space, where it is independently applied to the $R, G$ and $B$ colour channels. The same input image in the $R G B$ colour space is then transformed into HSV colour space. Following the same procedure, the CLAHE is applied to the $S$ and $V$ channels of the image, and later the channels will be merged and transformed back into $R G B$ colour space. The pixels' distribution is set according to the Rayleigh distribution for the CLAHE process in both $R G B$ and $H S V$ colour spaces. Such approaches of applying CLAHE in both colour spaces generate two separate images, namely CLAHE-RGB and CLAHE-HSV, where these images are combined using 
Euclidean norm to produce a noise-reduction and contrastenhanced image.

Abdul Ghani and Mat Isa (2014, 2015b) proposed methods integrating the modification of image histogram in $R G B$ and $H S V$ colour spaces. To meet the Rayleigh distribution, the histograms of each colour channel in $R G B$ colour space are improved and remapped. After that, the image is transformed into $H S V$ colour space with $S$ and $V$ channels are adjusted to a certain extent to minimize the impact of over- and underenhanced in the output image. Additionally, they (Abdul Ghani \& Mat Isa, 2015) suggested a two-stage approach that integrating the global and local contrast correction, as shown in Figure 5 . The goals of the proposed method are to increase image information and improve the brightness of underwater images while enhancing image contrasts. In summary, the proposed method has two major steps which are contrast and colour corrections. The dual-intensity images that generated by global contrast correction are therefore merged to produce contrast-enhanced images. Consequently, these images are processed locally to increase image information as well as to enhance saturation and brightness by correcting the colour of the images.

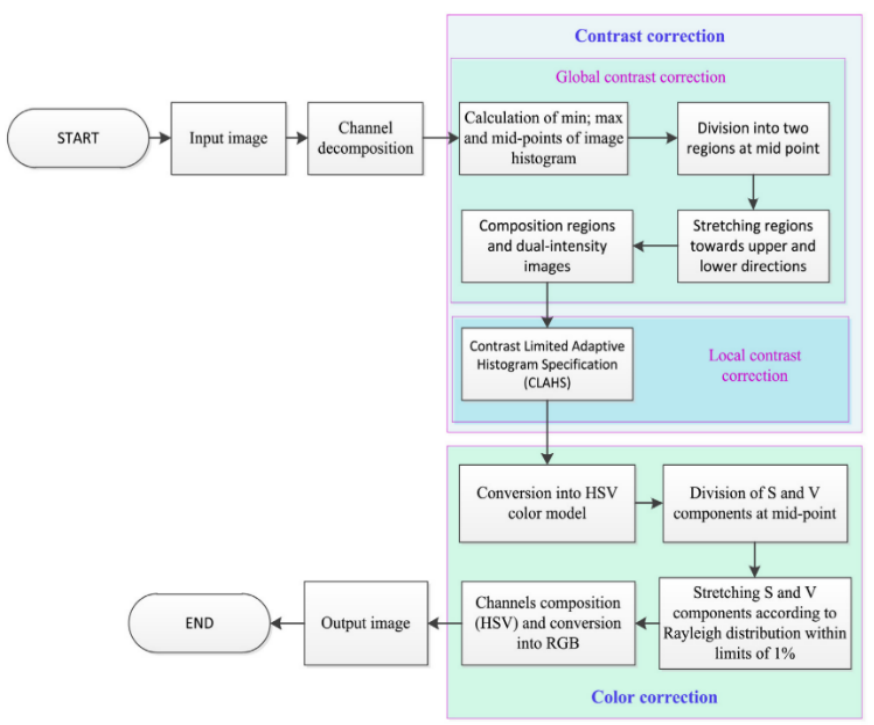

Figure 5. The flowchart of the proposed DIRS-CLAHS method by Abdul Ghani and Mat Isa (2015a)

Bianco et al. (2015) suggested an underwater color correction approach in the Ruderman opponent color space $l \alpha \beta$ based on Gray World (GW) with uniform lighting hypothesis. Color correction is achieved by white balance of chromatic components ( $\alpha$ and $\beta$ ), whereas the luminance component $l$ is processed to improve the image contrast. In addition, by developing the mathematical formulation of hue correction recommended by Reinhard et al. (2001), they have addressed the colour correction issue using a computational colour constancy method.

Moreover, Ancuti et al. (2012) proposed a method focused on the concept of fusion for improving the images and videos collected under various lighting conditions. Using a white balancing technique, colour correction is achieved to eliminate the colour casts and restore the gray and white shades of the image based on GW hypothesis. In addition, Ancuti et al. (2018) refined the technical execution of the fusion method by introducing an alternative and simpler input definitions and associated weight maps to improve the image quality. Figure 6 shows the summary method of combining two images, based on a (standard) multiscale fusion algorithm, from a white-balanced version of the single input.

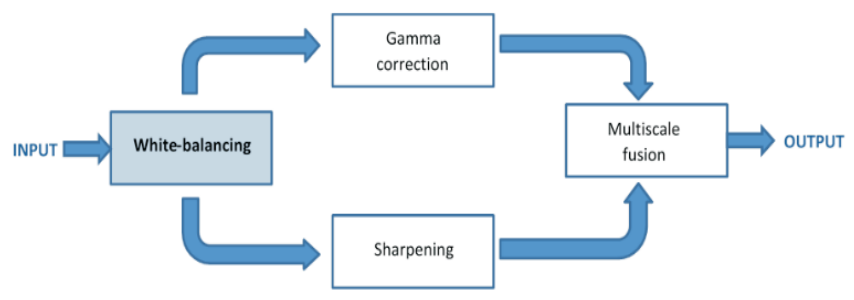

Figure 6. Method overview of Ancuti et al. (2018): two images are extracted from a white-balanced version of the single input, and are fused based on a multiscale fusion algorithm

Furthermore, Wong et al. (2019) suggested an integrated approach for removing the colour cast and improving the colourfulness and contrast of the underwater image. After the red and blue colour channels in the underwater have been compensated as recommended by Ancuti et al. (2018), the colour cast is eliminated by applying Adaptive GW (AGW) method, which is an adaptive variant of the GW method. The calculation of AGW method involves the global mean as well as the local mean of each channel of an image, and both are weighted before combining them. Thereafter, the Differential Gray-Levels Histogram Equalization for Color Images (DHECI) is applied to improve the underwater image's contrast and colourfulness. Figure 7 shows the flowchart of the Wong et al. (2019) sequential structure method. 


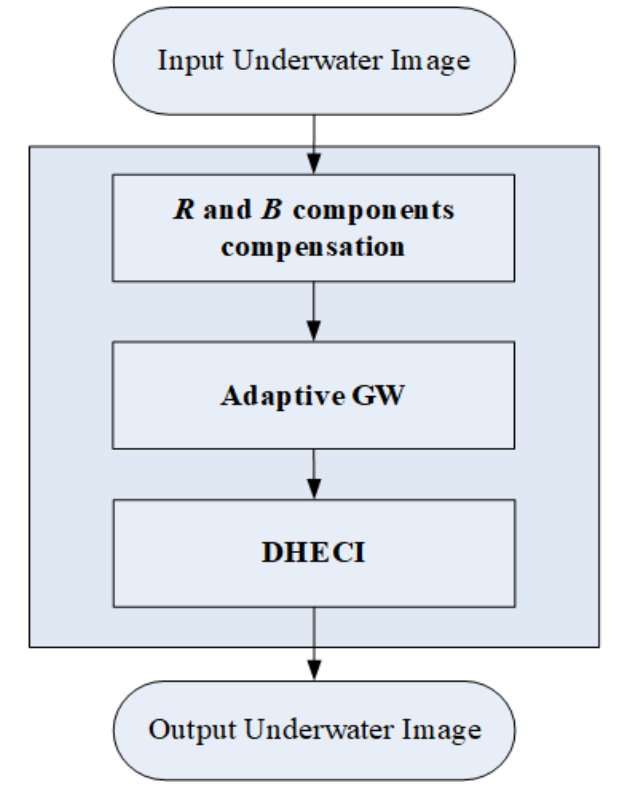

Figure 7. The flowchart of the Wong et al., (2019) method

\section{RESULT AND DISCUSSION}

Various different image quality assessment methods have been implemented and researched over the past couple of years, with the aim of creating a quality metric that incorporates perceptual quality measures by taking into account the characteristics of Human Visual System (HVS). The objective image quality metrics are divided in three groups, which are full-reference, no-reference and reduced-reference. Fullreference refers to where an original image is compared with the distorted image; no-reference may refer to the blind quality assessment where there is no reference image to be compared; whereas reduced-reference refers to which the reference image is only partially accessible in the form of extracted features set. Wang and Bovik (2006) have given a detailed review of the image quality assessments. The existing state-of-the-art methods of Carlevaris-Bianco et al. (2010), Drews-Jr et al. (2013), Emberton et al. (2015), Galdran et al. (2015), Li, Guo, Chen, et al. (2016), Ancuti et al. (2018), and Wong et al. (2019) are compared and analysed.

\section{A. Comparative Analysis using Qualitative Evaluations}

In the current state of underwater image processing, there is no ground truth image taken with standard illumination available that can be used to compare and evaluate it with any enhanced images. Thus, most of the cited methods for enhancement and restoration are not only relied on quantitative scores but also on visual inspection. Input images (Carlevaris-Bianco et. al., 2010; Ancuti et al., 2018) that were captured in different locations with different water environments, objects and backgrounds have been chosen for comparative analysis.

Figure 8 shows the visual comparative results of eight input images and their respective enhanced images using the stateof-the-art methods. As can be seen in all the eight source underwater images, they compose of haze, color cast, poor visibility and lack of contrast. From the visual inspection, some bluish and greenish casts still stay in the resultant images that are processed with the methods of CarlevarisBianco et al. (2010), Drews-Jr et al. (2013), Emberton et al. (2015), and Li, Guo, Chen, et al. (2016), because their methods concentrate more on dehazing the underwater scenes. It is noticeable that the methods of Galdran et al. (2015) and Ancuti et al. (2018) have constantly eliminated the bluish and greenish casts in the underwater images, but there are some enhanced areas which resulted in brownish or greyish colours left in the images. On the other hand, as demonstrated in Figure 8(h), the method of Wong et al. (2019) not only eliminated the unwanted colour cast and improved the visibility, it also has improved the brightness and preserved the natural appearance of the underwater images. This can be further validated by the quantitative evaluations that are shown in the tables in the next subsection. The overall resultant images produced by Wong et al. (2019) showed an improvement in terms of image color and contrast. The subjects and objects in the underwater images are well-observed as the color of the objects look more natural and pleasant, hence, leaving the viewers with a better impression. 


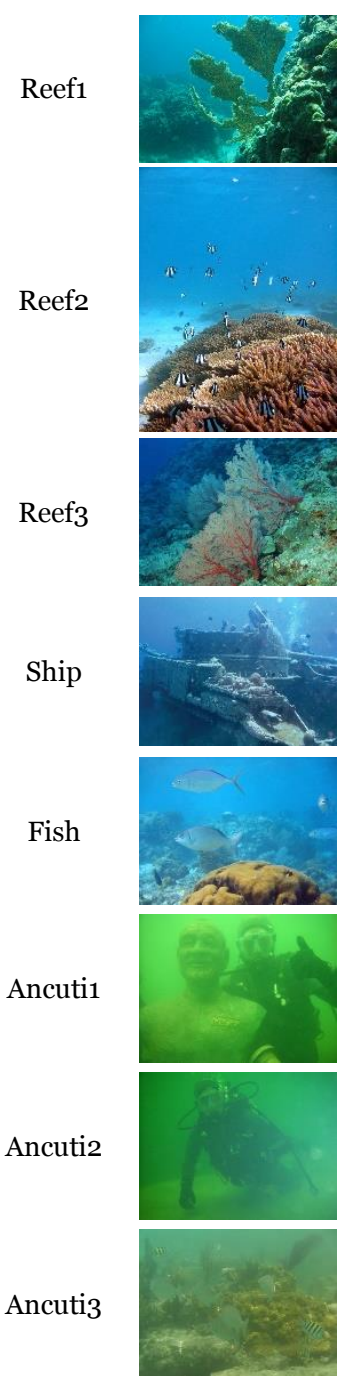

(a)
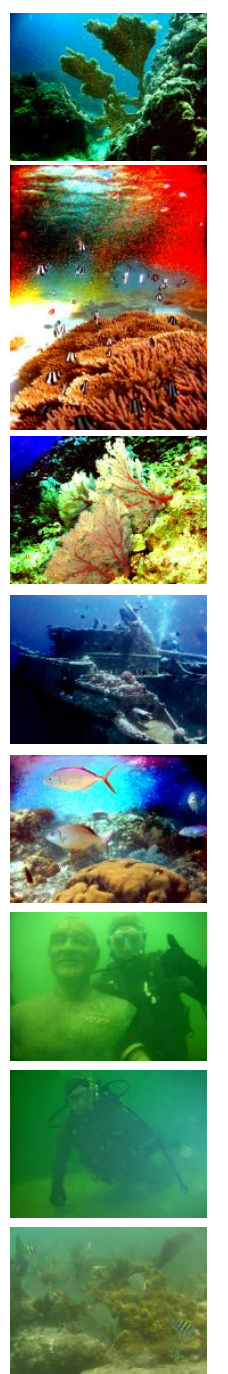

(b)
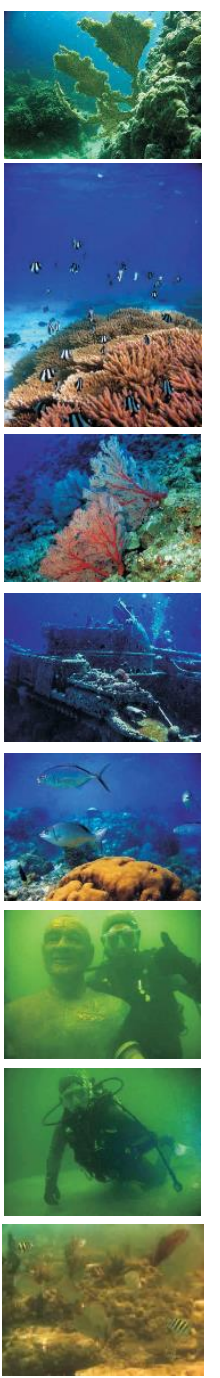

(c)
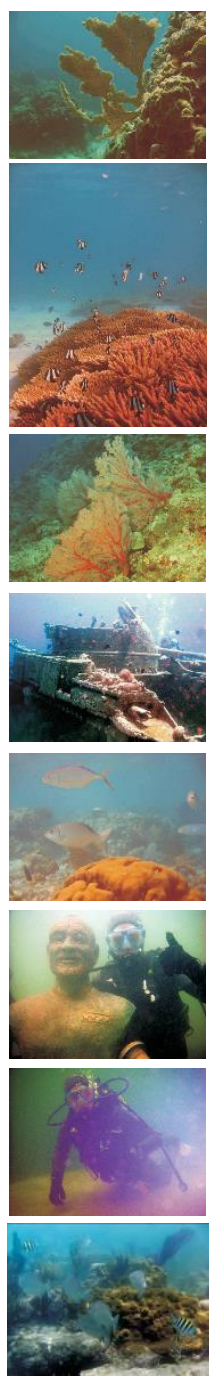

(d)
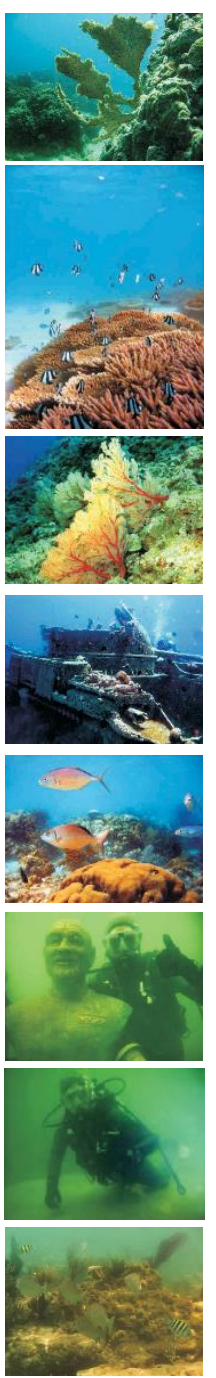

(e)

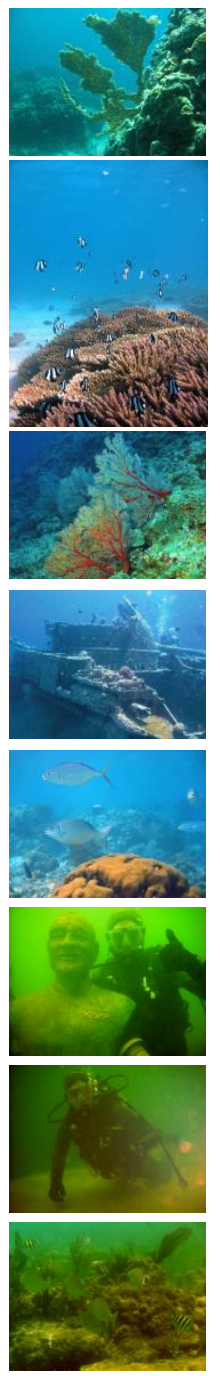

(f)
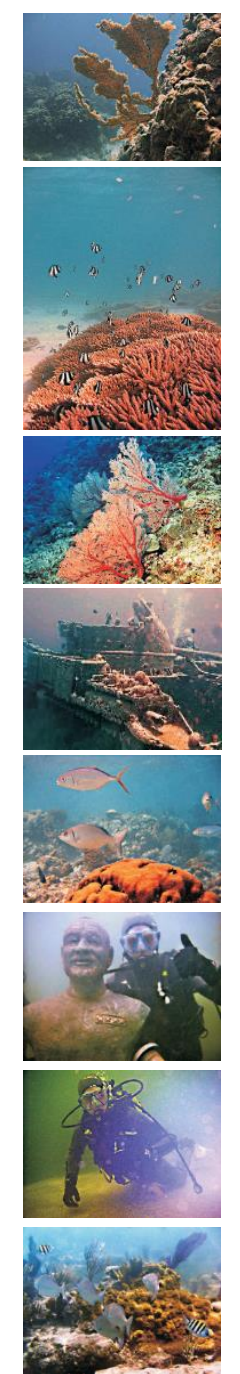

(g)

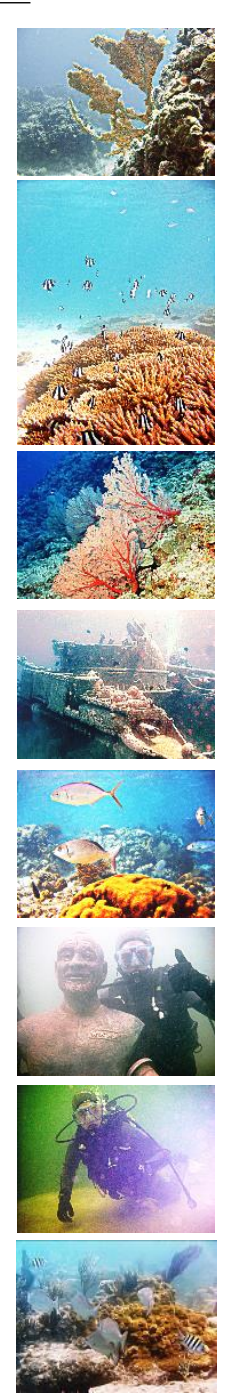

(h)

Figure 8. Visual comparative results of the state-of-the-art methods. (a) source images and their enhanced images after processing with the (b) Carlevaris-Bianco et al., (2010) method, (c) Drews-Jr et al., (2013) method, (d) Galdran et al., (2015) method, (e) Emberton et al., (2015) method, (f) Li, Guo, Chen, et al., (2016) method,

(g) Ancuti et al., (2018) method, and (h) Wong et al., (2019). The associated quantitative evaluation of these images is given in Tables 1-2. 
Table 1. Quantitative results based on UCIQE metric

\begin{tabular}{c|c|c|c|c|c|c|c|c}
\hline & $\begin{array}{c}\text { Source } \\
\text { image }\end{array}$ & $\begin{array}{c}\text { Carlevaris- } \\
\text { Bianco et } \\
\text { al., (2010) }\end{array}$ & $\begin{array}{c}\text { Drews-Jr } \\
\text { et al., } \\
\text { (2013) }\end{array}$ & $\begin{array}{c}\text { Galdran et } \\
\text { al., (2015) }\end{array}$ & $\begin{array}{c}\text { Emberton } \\
\text { et al., } \\
(\mathbf{2 0 1 5})\end{array}$ & $\begin{array}{c}\text { Li, Guo, } \\
\text { Chen, et } \\
\text { al., (2016) }\end{array}$ & $\begin{array}{c}\text { Ancuti et } \\
\text { al., (2018) }\end{array}$ & $\begin{array}{c}\text { Wong et., (2019) } \\
\text { al }\end{array}$ \\
\hline Reef1 & 0.58 & 0.74 & 0.65 & 0.58 & 0.66 & 0.57 & 0.66 & 0.74 \\
Reef2 & 0.65 & 0.71 & 0.66 & 0.63 & 0.72 & 0.62 & 0.71 & 0.73 \\
Reef3 & 0.52 & 0.75 & 0.62 & 0.53 & 0.68 & 0.52 & 0.70 & 0.74 \\
Ship & 0.55 & 0.81 & 0.55 & 0.65 & 0.63 & 0.55 & 0.63 & 0.74 \\
Fish & 0.53 & 0.75 & 0.62 & 0.53 & 0.71 & 0.53 & 0.67 & 0.70 \\
Ancuti1 & 0.43 & 0.46 & 0.50 & 0.64 & 0.50 & 0.46 & 0.59 & 0.74 \\
Ancuti2 & 0.41 & 0.43 & 0.49 & 0.53 & 0.53 & 0.44 & 0.59 & 0.69 \\
Ancuti3 & 0.42 & 0.44 & 0.54 & 0.61 & 0.56 & 0.46 & 0.66 & 0.73 \\
\hline Average & 0.51 & 0.63 & 0.58 & 0.59 & 0.62 & 0.52 & 0.65 & 0.73 \\
\hline
\end{tabular}

Table 2. Quantitative results based on UIQM metric

\begin{tabular}{c|c|c|c|c|c|c|c|c}
\hline & $\begin{array}{c}\text { Source } \\
\text { image }\end{array}$ & $\begin{array}{c}\text { Carlevaris- } \\
\text { Bianco et } \\
\text { al., (2010) }\end{array}$ & $\begin{array}{c}\text { Drews-Jr } \\
\text { et al., } \\
\text { (2013) }\end{array}$ & $\begin{array}{c}\text { Galdran et } \\
\text { al., (2015) }\end{array}$ & $\begin{array}{c}\text { Emberton } \\
\text { et al., } \\
\text { (2015) }\end{array}$ & $\begin{array}{c}\text { Li, Guo, } \\
\text { Chen, et } \\
\text { al., (2016) }\end{array}$ & $\begin{array}{c}\text { Ancuti et } \\
\text { al., (2018) }\end{array}$ & $\begin{array}{c}\text { Wong et., (2019) } \\
\text { al }\end{array}$ \\
\hline Reef1 & 0.75 & 0.86 & 0.66 & 0.57 & 0.69 & 0.71 & 0.69 & 0.61 \\
Reef2 & 0.85 & 0.81 & 0.65 & 0.67 & 0.76 & 0.81 & 0.78 & 0.86 \\
Reef3 & 0.73 & 0.64 & 0.58 & 0.52 & 0.68 & 0.71 & 0.77 & 0.75 \\
Ship & 0.48 & 0.72 & 0.49 & 0.61 & 0.59 & 0.48 & 0.67 & 0.61 \\
Fish & 0.68 & 0.88 & 0.57 & 0.53 & 0.76 & 0.67 & 0.62 & 0.83 \\
Ancuti1 & 0.35 & 0.52 & 0.38 & 0.46 & 0.41 & 0.59 & 0.51 & 0.64 \\
Ancuti2 & 0.37 & 0.50 & 0.34 & 0.53 & 0.43 & 0.60 & 0.69 & 0.81 \\
Ancuti3 & 0.44 & 0.51 & 0.49 & 0.65 & 0.56 & 0.67 & 0.65 & 0.73 \\
\hline Average & 0.58 & 0.68 & 0.52 & 0.57 & 0.61 & 0.65 & 0.67 & 0.73 \\
\hline
\end{tabular}

\section{B. Comparative Analysis using Quantitative Evaluations}

Previously, limited underwater image quality metrics can be applied to choose good quality images in different marine habitats. Objective assessment is difficult in this case as it requires the availability of ground truth and therefore, noreference metrics are needed. Recently, there are two quantitative measurements have been developed specifically for underwater image processing, which is Underwater Color Image Quality Evaluation (UCIQE) (Yang \& Sowmya, 2015), which is used to quantify the non-uniform colour cast, blurring and low contrast of underwater images, and Underwater Image Quality Measure (UIQM) (Panetta, Gao \& Agaian, 2016), which is used to measure the underwater image colourfulness, sharpness and contrast.

The quantitative performance evaluations of UCIQE and
UIQM for Figure 8 are summarized in Tables 1 - 2, respectively. The values in bold and italic represent the highest and second highest scores, respectively. As shown in the tables, the method of Wong et al. (2019) gave the highest average UCIQE and UIQM scores when compared to the other state-of-the-art methods listed in the tables. These demonstrated that the method not only can effectively increase the information and visibility of the underwater images, they can restore the well-balanced chroma, saturation, sharpness as well as contrast of the enhanced underwater images.

\section{CONCLUSION}

In conclusion, the light scattering and light absorption are the main causes in the underwater environment, which causes the images to be hazy, blurry, colour cast and poor 
contrast. As discussed, the underwater image processing can be addressed from two different contexts. This paper reviews the underwater image restoration and image enhancement methods, where these methods are able to remove the color cast and haziness on the images as well as to improve the image colourfulness and contrast. Generally, underwater image restoration refers to the improvement of underwater image quality using a model of the degradation and the original image formation, while the image enhancement methods are performed on the image itself without prior knowledge. The characteristics of each of the developed methods by the researchers are highlighted, and their performances are evaluated both subjectively and objectively by the quality assessment methods.

\section{ACKNOWLEDGEMENT}

The author would like to thank the anonymous reviewers for their valuable comments and suggestion on this paper.

\section{REFERENCES}

Abdul Ghani, AS 2015, 'Improvement of underwater image contrast enhancement technique based on histogram modification', $\mathrm{PhD}$ thesis, University Sains Malaysia, Malaysia.

Abdul Ghani, AS \& Mat Isa, NA 2014, 'Underwater image quality enhancement through Rayleigh-stretching and averaging image planes', International Journal of Naval Architecture and Ocean Engineering, vol. 6, no. 4, pp. 840866.

Abdul Ghani, AS \& Mat Isa, NA 2015, 'Enhancement of low quality underwater image through integrated global and local contrast correction', Applied Soft Computing, vol. 37, pp. 332-344.

Abdul Ghani, AS \& Mat Isa, NA 2015, 'Underwater image quality enhancement through integrated color model with Rayleigh distribution', Applied Soft Computing, vol. 27, pp. 219-230.

Abdul Ghani, AS \& Mat Isa, NA 2017, 'Automatic system for improving underwater image contrast and color through recursive adaptive histogram modification', Computers and Electronics in Agriculture, vol. 141, pp. 181-195.

Akila, C \& Varatharajan, R 2018, 'Color fidelity and visibility enhancement of underwater image de-hazing by enhanced fuzzy intensification operator', Multimedia Tools and Applications, Multimedia Tools and Applications, vol. 77, no. 4, pp. 4309-4322.

Ancuti, C, Ancuti, CO, Haber, T \& Bekaert, P 2012, 'Enhancing underwater images and videos by fusion', in 2012 IEEE Conference on Computer Vision and Pattern Recognition, June 2012, Providence, RI, USA.

Ancuti, CO, Ancuti, C, De Vleeschouwer, C \& Bekaert, P 2017, 'Color balance and fusion for underwater image enhancement', IEEE Transactions on image processing, vol. 27, no. 1, pp. 379-393.

Anthoni, JF 2012, Water and light in underwater photography, viewed 5 June 2018, http://www.seafriends.org.nz/phgraph/water.htm.

Ao, J \& Ma, C 2018, 'Adaptive stretching method for underwater image color correction', International Journal of Pattern Recognition and Artificial Intelligence, vol. 32, no. 2, pp. 1854001

Bianco, G, Muzzupappa, M, Bruno, F, Garcia, R \& Neumann, L 2015, 'A new color correction method for underwater imaging', The International Archives of Photogrammetry, Remote Sensing and Spatial Information Sciences, vol. 40, no. 5 , pp. 25-32.

Carlevaris-Bianco, N, Mohan, A \& Eustice, RM 2010, 'Initial results in underwater single image dehazing', in Oceans 2010 Mts/Ieee Seattle, September 2010, Seattle, WA, USA.

Chambah, M, Semani, D, Renouf, A, Courtellemont, P \& Rizzi, A 2003, 'Underwater color constancy: enhancement of automatic live fish recognition', in Color Imaging IX: Processing, Hardcopy, and Applications, International Society for Optics and Photonics, 18 December 2003, San Jose, California, United States.

Chao, L \& Wang, M 2010, 'Removal of water scattering', in 2nd International Conference on Computer Engineering and Technology, April 2010, Chengdu, China.

Chiang, JY \& Chen, Y-C 2012, 'Underwater image enhancement by wavelength compensation and dehazing', IEEE Transactions on Image Processing, vol. 21, no. 4, pp. $1756-1769$.

Drews, P, Nascimento, E, Moraes, F, Botelho, S \& Campos, M 2013, 'Transmission estimation in underwater single 
images', in Proceedings of the IEEE international conference on computer vision workshops, December 2013, Darling Harbour, Sydney.

Emberton, S, Chittka, L \& Cavallaro, A 2015, 'Hierarchical rank-based veiling light estimation for underwater dehazing', in Procedings of the British Machine Vision Conference 2015, British Machine Vision Association, September 2015, Swansea University Singleton Campus, Swansea, UK.

Fattal, R 2008, 'Single image dehazing', ACM Transactions on Graphics, vol. 27, no. 3, pp. 1.

Galdran, A, Pardo, D, Picón, A \& Alvarez-Gila, A 2015, 'Automatic red-channel underwater image restoration', Journal of Visual Communication and Image Representation, vol. 26, pp. 132-145.

Ghani, ASA \& Isa, NAM 2015, 'Homomorphic filtering with image fusion for enhancement of details and homogeneous contrast of underwater image', Indian Journal of Geo-Marine Sciences, vol. 44, no. 12, pp. 1904-1919.

Gonzalez, RC \& Woods, RE 2008, Digital image processing third edition, Pearson Prentice Hall, New Jersey, USA.

Gorman, GA 2011, 'Field deployable dynamic lighting system for turbid water imaging', PhD thesis, Massachusetts Institute of Technology, USA.

Gupta, M, Narasimhan, SG \& Schechner, YY 2008, 'On controlling light transport in poor visibility environments', in 2008 IEEE Conference on Computer Vision and Pattern Recognition, June 2008, Anchorage, AK, USA.

Hautiere, N, Tarel, JP, Aubert, D \& Dumont, E 2008, 'Blind contrast enhancement assessment by gradient ratioing at visible edges', Image Analysis \& Stereology, vol. 27, no. 2, pp. 87-95.

He, K, Sun, J \& Tang, X 2009, 'Single image haze removal using dark channel prior', in 2009 IEEE Conference on Computer Vision and Pattern Recognition, 2009, Miami, Florida, USA.

He, K, Sun, J, \& Tang, X 2011, 'Single image haze removal using dark channel prior', IEEE Transactions on Pattern Analysis and Machine Intelligence, vol. 33, no, 12, pp. 2341-2353.

Hitam, MS, Awalludin, EA, Yussof, WNJHW \& Bachok, Z 2013, 'Mixture contrast limited adaptive histogram equalization for underwater image enhancement', in 2013 International conference on computer applications technology (ICCAT), January 2013, Sousse, Tunisia.

Iqbal, K, Salam, RA, Osman, A \& Talib, AZ 2007, 'Underwater image enhancement using an integrated colour model', IAENG International Journal of computer science, vol. 34, no. 2.

Iqbal, K, Odetayo, M, James, A, Salam, RA \& Talib, AZH 2010,
'Enhancing the low quality images using unsupervised colour correction method', in 2010 IEEE International Conference on Systems, Man and Cybernetics, October 2010, Istanbul, Turkey.

Jaffe, JS 1990, 'Computer modeling and the design of optimal underwater imaging systems', Journal of Oceanic Engineering, vol. 15, no. 2, pp. 101-111.

Jaffe, JS 2010, 'Enhanced extended range underwater imaging via structured illumination', Optics Express, vol. 18, no. 12, pp. 12328-12340.

Kaeli, JW 2013, 'Computational strategies for understanding underwater optical image datasets', PhD thesis, Massachusetts Institute of Technology, USA.

Li, CY, Guo, JC, Cong, RM, Pang, YW \& Wang, B 2016, 'Underwater image enhancement by dehazing with minimum information loss and histogram distribution prior', IEEE Transactions on Image Processing, vol. 25, no. 12 , pp. 5664-5677.

Li, C, Guo, J, Chen, S, Tang, Y, Pang, Y \& Wang, J 2016, 'Underwater image restoration based on minimum information loss principle and optical properties of underwater imaging', in 2016 IEEE International Conference on Image Processing (ICIP), September 2016, Phoenix, AZ, USA.

McGlamery, BL 1980, 'A computer model for underwater camera systems', Ocean Optics VI, vol. 208, pp. 221-231.

Panetta, K, Gao, C \& Agaian, S 2016, 'Human-visualsystem-inspired underwater image quality measures', IEEE Journal of Oceanic Engineering, vol. 41, no. 3, pp. 541-551.

Peng, Y-T \& Cosman, PC 2017, 'underwater image restoration based on image blurriness and light absorption', IEEE Transactions on Image Processing, vol. 26, no. 4, pp. 1579-1594.

Reinhard, E, Adhikhmin, M, Gooch, B \& Shirley, P 2001, 'Color transfer between images', IEEE Computer graphics and applications, vol. 21, no. 5, pp. 34-41.

Schechner, YY \& Karpel, N 2004, 'Clear underwater vision', in Proceedings of the 2004 IEEE Computer Society Conference on Computer Vision and Pattern Recognition, June 2004, Washington, DC, USA.

Schettini, R \& Corchs, S 2010, 'Underwater image processing: state of the art of restoration and image enhancement methods', EURASIP Journal on Advances in Signal Processing, vol. 2010, no. 1, p. 746052.

Singh, H, Roman, C, Pizarro, O, Eustice, R \& Can, A 2007, 'Towards high-resolution imaging from underwater 
vehicles', The International Journal of Robotics Research, vol. 26 , no. 1 , pp. 55-74.

Torres-Mendez, LA \& Dudek, G 2005, 'Color correction of underwater images for aquatic robot inspection', in Energy Minimization Methods in Computer Vision and Pattern Recognition, November 2005, Springer, Berlin, Heidelberg.

Treibitz, T \& Schechner, YY 2009, 'Active polarization descattering', IEEE Transactions on Pattern Analysis and Machine Intelligence, vol. 31, no. 3, pp. 385-399.

Vasilescu, I, Detweiler, C \& Rus, D 2011, 'Color-accurate underwater imaging using perceptual adaptive illumination', Autonomous Robots, vol. 31, no. 2-3, pp. 285-296.

Wang, Z \& Bovik, AC 2006, 'Modern image quality assessment', Synthesis Lectures on Image, Video, and Multimedia Processing, vol. 2, no. 1, pp. 1-156.

Wong, SL, Paramesran, R, Yoshida, I \& Taguchi, A 2019, 'An integrated method to remove color cast and contrast enhancement for underwater image', IEICE Transactions on Fundamentals of Electronics, Communications and Computer Sciences, vol. 102, no. 11, pp. 1524-1532.

Wong, S-L, Paramesran, R \& Taguchi, A 2018, 'Underwater image enhancement by adaptive gray world and differential gray-levels histogram equalization', Advances in Electrical and Computer Engineering, vol. 18, no. 2, pp. 109-116.

Xie, K, Pan, W \& Xu, S 2018, 'An underwater image enhancement algorithm for environment recognition and robot navigation', Robotics, vol. 7, no. 1, pp. 14.

Yang, M \& Sowmya, A 2015, 'An underwater color image quality evaluation metric', IEEE Transactions on Image Processing, vol. 24, no. 12, pp. 6062-6071. 